\title{
PCORnet-defined Condition Algorithm
}

National Cancer Institute

\section{Source}

National Cancer Institute. PCORnet-defined Condition Algorithm. NCI Thesaurus. Code C154404.

An algorithm designed to identify health conditions as defined by the National PatientCentered Clinical Research Network. 